\title{
Effect of the Flow Channel Structure on the Nanofiltration Separation Performance
}

\author{
Zhi Chen, Ben Zhao, Fanglei Chen, and Jianming Li \\ School of Chemical Engineering, Sichuan University, Sichuan 610065, China \\ Correspondence should be addressed to Jianming Li; lijianming@scu.edu.cn
}

Received 27 January 2013; Accepted 20 March 2013

Academic Editor: Yongcheng Jin

Copyright (C) 2013 Zhi Chen et al. This is an open access article distributed under the Creative Commons Attribution License, which permits unrestricted use, distribution, and reproduction in any medium, provided the original work is properly cited.

\begin{abstract}
Two kinds of newly designed feed channels, for example, a spiral and a serpentine feed channels, for a bench-scale nanofiltration module were developed to improve the filtration performance. The experiments were carried out with the modules using a commercial flat NF membrane to investigate the effects of Reynolds number (Re) and flow channel structures on the flux of permeate and $\mathrm{Mg}^{2+}$ rejection. It was shown from the experimental results that although the effects of Reynolds number on fluxes were not obvious for the two new feed channels compared with a normal flow channel structure, the $\mathrm{Mg}^{2+}$ rejections varied apparently with Re. The $\mathrm{Mg}^{2+}$ rejections were almost the same for the modules with two new feed channels and larger than that for the module with normal feed channel. The numerical simulations of fluid flow in the three kinds of feed channels were completed at Re of 4800 to explain the phenomena. The results demonstrated that there was a secondary flow in both new feed channels, which strongly influences the $\mathrm{Mg}^{2+}$ rejection. The rejection increased with increasing average shear stress at the membrane wall. The spiral feed channel was the best one among the flow channel structures investigated.
\end{abstract}

\section{Introduction}

Nanofiltration is an intermediate filtration process between reverse osmosis (RO) and ultrafiltration (UF) that rejects molecules having a size of about one nanometer [1]. It has been introduced since 1980s, mainly used for softening water and removing organics. It has found many applications in various water purification and treatment as well as product separation processes because of its two remarkable features: one is the molecular weight cut-off (MWCO) which ranges from 200 to $2000 \mathrm{Da}$; the other is the separation of electrolytes due to the membrane materials containing charged groups $[2,3]$. Lots of studies on NF membrane have been carried out based on these features in the recent years, and it becomes the emphasis how to improve its separation performance.

The investigations mainly focus on two aspects: one is to develop new NF membrane materials with good separation performance; another is to improve operation conditions of NF process based on separation mechanism and modeling. $\mathrm{Ba}$ et al. fabricated stable NF membrane by chemical modification of P84 copolyimide asymmetric membranes using branched polyethylenimine (PEI) [4]. The membrane could be used in severe operating environments including high temperature $\left(100^{\circ} \mathrm{C}\right)$ and organic solvents. Jahanshahi et al. prepared two kinds of polymer composite NF membranes, that is, poly(piperazine-amide) and poly(vinyl alcohol) NF membranes, with good stability of structure and performance through a series of experiments [5]. Abu Seman et al. used the hydrophilic anionic monomer acrylic acid and the immersion method to modify a commercial polyethersulfone NF membrane by UV-initiated graft polymerization technique [6]. The membranes showed lower fouling tendency than the unmodified membrane. Ballet et al. studied experimentally the effects of feed pressure, ionic strength, concentration, and $\mathrm{pH}$ on the retention of phosphate anions [7]. Li et al. investigated the effects of pressure, flow rate on flux, and retention in seawater desalination process [8]. Pang carried out a research on the influences of pressure, temperature, influent flow, and $\mathrm{pH}$ on flux and desalination rate for the application of wastewater treatment in salt chemical plant [9]. Darvishmanesh et al. developed a new semiempirical model based on the traditional solution diffusion with imperfection model for solvent resistant nanofiltration [10]. The new model demonstrated a good prediction for the flux of solvent 
through the nanofiltration. Fadaei et al. developed a mass transfer model to predict ion transport through the NF membrane account for the concentration polarization phenomenon and its influence on the ion separation [11]. They solved model equations numerically using computational fluid dynamics (CFD) techniques and successfully predicted the local concentration of ions, permeate flux, and rejection of ions in a rectangular crossflow NF membrane module. Wang et al. review the research progresses on the evaluation of pore structure and electrical property, the separation mechanism and modeling, and the electrokinetic phenomena of the NF membranes over the past two decades [12].

Furthermore, there have been investigations of flow patterns and mass transfer in NF membrane module channels using fluid dynamics for recent years [11, 13-17]. The wall shear stress could be increased by placing flow-aligned spacers $[13,17]$ or feed spacers [15] or utilizing vibration [14] to increase the filtration performances. However, there were few studies aiming at the influence of flow channel structures of membrane modules on the separation performance of NF membrane.

In this article, a bench-scale modules, with two kinds of new designed flow channel structures, that is, a spiral flow channel and a serpentine flow channel ones as well as a normal one, were used for experiments in order to investigate the flux and $\mathrm{Mg}^{2+}$ rejection of a commercial NF membrane by changing flow pattern from laminar to turbulent flow regime in the flow channel. Moreover, the numerical simulations of fluid flow in the modules with the three kinds of feed channels were completed at Reynolds number of 4800 terms of CFD techniques to observe the effects of secondary flow in curved flow duct and wall shear stress on $\mathrm{Mg}^{2+}$ rejection.

\section{Numerical Simulation}

2.1. Dean Vortex. A couple of reverse symmetric vortices which are named Dean vortex [18] generate because of secondary flow formed by centrifugal force when incompressible fluid flows in curved flow duct because the fluid in the faster core, as a result of centrifugal effects, is directed outward and, in order to satisfy continuity, the slower fluid near the boundaries is directed inward. The disturbance on membrane surface enhanced by Dean vortex will hinder concentration polarization development and membrane fouling effectively in the membrane separation process. Dean pointed out that Dean vortex generate as the result of the interaction of centrifugal force with viscous force borne by fluid in the pipe; thus, a dimensionless number which is called Dean number is defined as the ratio of centrifugal force to viscous force [19]. Fluid falls into unstable state when Dean number is above a critical value. In this paper, the numerical simulation of secondary flow was completed based on CFD technology in order to investigate the effects of Dean vortex on the flux and salt rejection.

2.2. Governing Equations. There are swirling flows when fluid flows in curved duct, such as spiral flow channel and the serpentine flow channel, in the module. Therefore, realizable $k$ - $\varepsilon$ turbulence model was employed to simulate secondary flows, and empirical constants $C_{\mu}$ and $C_{1}$ in standard $k$ $\varepsilon$ model were supposed as variables. Water was used as fluid medium in the numerical simulation. For isothermal, incompressible, Newtonian, and a steady-state flow, the governing equations, consisting of continuity equation, momentum equation, turbulent kinetic energy $(k)$ equation, and turbulent dissipation rate $(\varepsilon)$ equation, may be written as follows [20, 21]:

$$
\begin{aligned}
\frac{\partial u_{i}}{\partial x_{i}} & =0 \\
\frac{\partial}{\partial x_{j}} & \left(\rho u_{j} u_{i}\right) \\
& =-\frac{\partial p}{\partial x_{i}}+\frac{\partial}{\partial x_{j}}\left[\left(\mu+\mu_{t}\right)\left(\frac{\partial u_{j}}{\partial x_{i}}+\frac{\partial u_{i}}{\partial x_{j}}\right)\right]+\rho g_{i},
\end{aligned}
$$

where $\rho$ is the liquid density, $u$ is the liquid velocity, $\mu$ is the dynamic viscosity, $p$ is the pressure, $g$ is the acceleration of gravity, and $\mu_{t}$ is the turbulent dynamic viscosity expressed as follows:

$$
\begin{gathered}
\mu_{t}=C_{\mu} \rho \frac{k^{2}}{\varepsilon}, \\
C_{\mu}=\frac{1}{A_{0}+A_{s} U^{*} k / \varepsilon}, \\
\frac{\partial}{\partial x_{j}}\left(\rho u_{j} k\right)=\frac{\partial}{\partial x_{j}}\left[\left(\mu+\frac{\mu_{t}}{\sigma_{k}}\right) \times \frac{\partial k}{\partial x_{j}}\right]+G_{k}-\rho \varepsilon, \\
\frac{\partial}{\partial x_{j}}\left(\rho \varepsilon u_{j}\right)=\frac{\partial}{\partial x_{j}}\left[\left(\mu+\frac{\mu_{t}}{\sigma_{\varepsilon}}\right) \times \frac{\partial \varepsilon}{\partial x_{j}}\right] \\
+\rho C_{1} S \varepsilon-\rho C_{2} \frac{\varepsilon^{2}}{k+\sqrt{\nu \varepsilon}},
\end{gathered}
$$

where the model constants are $\sigma_{k}=1.0, \sigma_{\varepsilon}=1.2, C_{2}=1.90$, and

$$
C_{1}=\max \left[0.43, \frac{\eta}{\eta+5}\right]
$$

2.3. Model Geometry. The membrane module used in experiments and numerical simulations was a conventional crossflow filtration one that had a flat circular cell with a feed chamber thickness of $14 \mathrm{~mm}$ and a diameter of $94 \mathrm{~mm}$. Flange structure was adopted for the module to divide the cell into two sides, which was convenient for frequent dismounting and cleaning. The upside cell was used as a feed channel and the downside cell was used to place a NF membrane. There were three types of feed channels. The normal feed channel was the upside cell without any inner deflector to direct flow. The spiral feed channel and serpentine feed channel were formed by welding deflectors as shown in Figure 1.

There are three computational domains corresponding to the three types of the modules. One is the normal feed channel that consists of cylindrical cell, inlet pipe, and outlet 


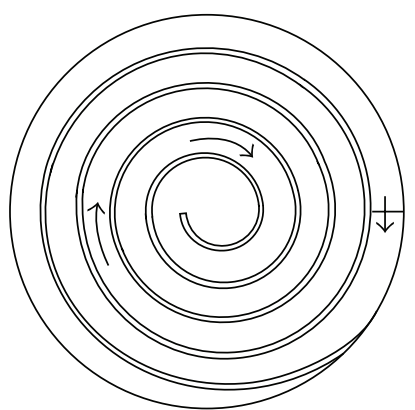

(a) Spiral flow channel

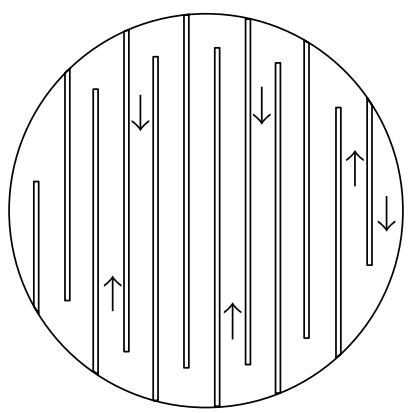

(b) Serpentine flow channel
FIGURE 1: Feed channels in the upside cells of the NF membrane modules.

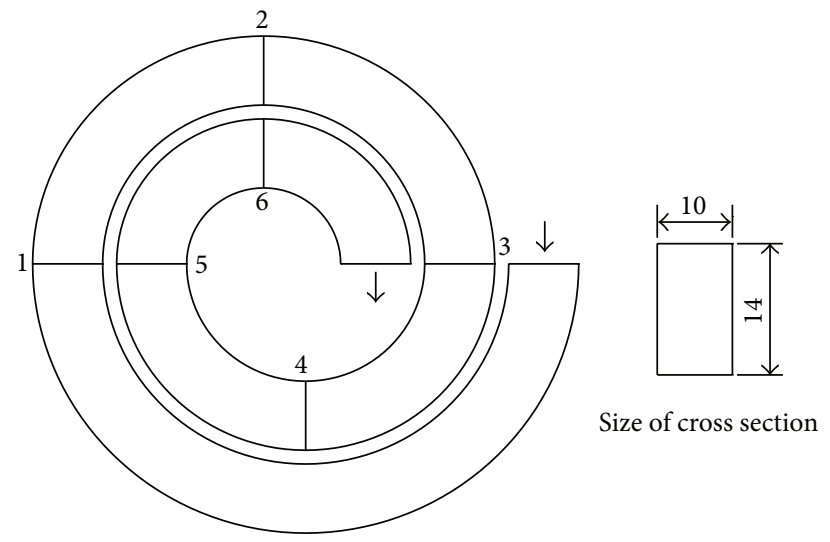

FIGURE 2: Schematic diagram of the model and section of the spiral flow channel.

pipe. The computational domain of the spiral feed channel, whose cross section size is $10 \mathrm{~mm} \times 14 \mathrm{~mm}$, is shown in Figure 2, and the computational domain of the serpentine feed channel is shown in Figure 3. The cross section size of the U-shaped duct is different from that of straight channel for the serpentine feed channel. The former is $15 \mathrm{~mm} \times 14 \mathrm{~mm}$, and the latter is $10 \mathrm{~mm} \times 14 \mathrm{~mm}$. The six cross sections are chosen to observe the velocity vector fields for both spiral and serpentine feed channels as shown in Figures 2 and 3.

2.4. Numerical Solution. The governing equations with the corresponding boundary conditions were numerically solved using FLUENT software. The software uses the finite volume method for numerical solution of model equations. The three-dimensional computational grid was generated using GAMBIT software package for each type of feed channel. The grid is an unstructured hexahedral grid with 261,203 grid points for the normal feed channel, 244,209 mesh nodes for the spiral feed channel, and 317,289 grid points for the serpentine feed channel. The consecutive simulations with the increasing number of grid points were performed until obtaining a grid-independent result. The pressure-velocity coupling SIMPLE scheme was used. The governing equations were discretized with the first-order upwind scheme. The operating temperature was of $35^{\circ} \mathrm{C}$, and the channel Reynolds

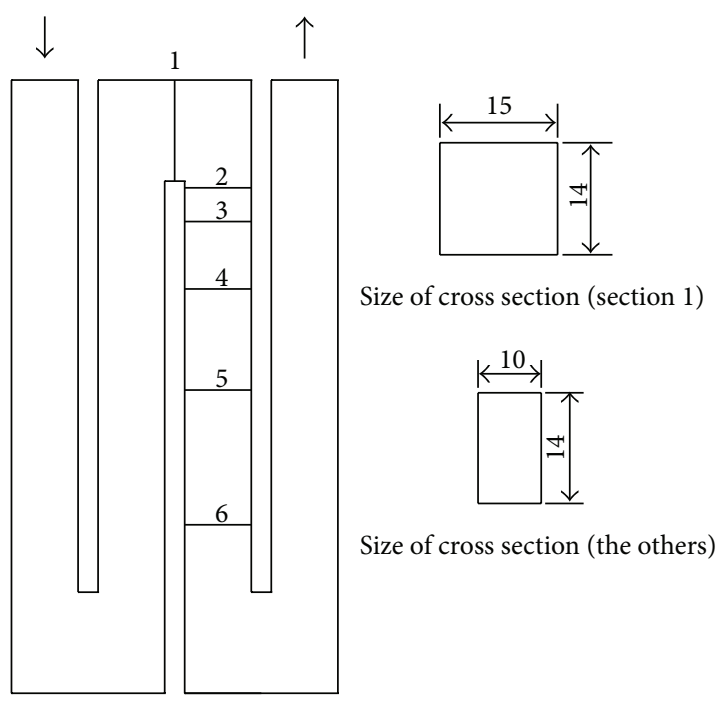

FIGURE 3: Schematic diagram of the model and sections of the serpentine flow channel.

number was 4800 in the numerical simulation. The inlet is rectangle pipe with the size of $10 \mathrm{~mm} \times 14 \mathrm{~mm}$ for each membrane module so that the inlet velocity of water could be of $0.301 \mathrm{~m} / \mathrm{s}$ for the spiral feed channel or the serpentine feed channel and $0.707 \mathrm{~m} / \mathrm{s}$ for the normal feed channel. Boundary conditions, such as velocity-inlet, out-flow, standard wall functions, stationary wall, and no slip conditions, were used in numerical simulation. The inlet turbulent intensity and hydraulic diameter were $2.84 \%$ and $12 \mathrm{~mm}$, respectively. The numerical simulation would continue until a relative tolerance set is less than $10^{-6}$.

\section{Experimental}

3.1. Apparatus. Main apparatuses and instruments used in the experiments (as shown in Figure 4) included a vortex pump (WB25-72), two glass rotameters (LZB-15F and LZB25F), an electronic balance (SL502N), a 1/10000 electronic balance (FA1204B), and two pressure gauges with the grade of 1.6 .

3.2. Membranes and Materials. The TFC-SR100 NF membrane was used in the experiments, and its molecular weight cut-off was about 200. Experimental reagents included ethylenediaminetetraacetic acid (EDTA), anhydrous alcohol $(0.789 \sim 0.791 \mathrm{~g} / \mathrm{mL})$, pure ammonia, ammonium chloride $\left(\mathrm{NH}_{4} \mathrm{Cl}\right)$, magnesium sulfate $\left(\mathrm{MgSO}_{4}\right)$, triethanolamine, and eriochrome black $\mathrm{T}$. All of them are analytical grade and used as received.

3.3. Method for Measuring $\mathrm{Mg}^{2+}$ Concentration. The solution of magnesium sulfate, in which $\mathrm{Mg}^{2+}$ concentration is $150 \mathrm{ppm}$ to simulate the water hardness of Yangtze River, was prepared with anhydrous magnesium sulfate and deionized water for NF experiments. EDTA complexometry [22] was used to measure $\mathrm{Mg}^{2+}$ concentration. The brief chemical 
equations of indicator, complex, and terminal reactions are described, respectively, as

$$
\begin{gathered}
\left.\mathrm{Mg}^{2+}+\text { EBT (eriochrome black } \mathrm{T}\right) \longrightarrow \mathrm{Mg}-\mathrm{EBT}(\text { wine red) } \\
\mathrm{Mg}^{2+}+\mathrm{Y}^{4-}(\text { EDTA }) \longrightarrow \mathrm{MgY}^{2-} \text { (colorless) }
\end{gathered}
$$$$
\mathrm{Mg} \text {-EBT (wine red) }+\mathrm{Y}^{4-} \longrightarrow \mathrm{MgY}^{2-}+\mathrm{EBT} \text { (pure blue) . }
$$

3.4. Nanofiltration Performance Tests. NF performance tests were conducted by a crossflow membrane module at different temperatures under different operating pressures, and the effective filtration area was $69.40 \mathrm{~cm}^{2}$. The test rig for nanofiltration is shown in Figure 4. At steady state, the weight of permeate collected in the triangular flask was measured by an electronic balance to calculate the total flux, $J_{v}$, as follows:

$$
J_{v}=\frac{W}{A \cdot t},
$$

where $W$ is the total mass permeated in the test time $t$ and $A$ is the effective area. The $\mathrm{Mg}^{2+}$ concentrations of feed and permeated samples were measured by the EDTA titration method for $\mathrm{MgSO}_{4}$ solution to calculate the $\mathrm{Mg}^{2+}$ rejection, $R_{\text {obs }}$ as follows:

$$
R_{\mathrm{obs}}=\frac{c_{b}-c_{p}}{c_{b}} \times 100 \% \text {, }
$$

in which $c_{b}$ and $c_{p}$ are the $\mathrm{Mg}^{2+}$ concentrations of feed and permeate, respectively.

In the experiment, feed solution in the feed tank was pumped to the membrane module by vortex pump. The permeate flowed through the membrane into the triangular flask, and the concentrate was then returned to feed tank. The operating pressure and crossflow velocity were kept constant by adjusting both the valve and the rotation speed of the pump. The temperature was regulated by controlling the flow rate of cooling water. Permeate flux was measured, and permeate sample collected at steady state that was assumed after $3 \mathrm{~h}$ of operation. The samples were analyzed in the method mentioned in Section 3.3. The volume of feed was of $5 \mathrm{~L}$ so that $\mathrm{Mg}^{2+}$ concentration of feed $c_{b}$ might be constant because the volume of permeate was relatively small.

Firstly, membrane module with normal flow channel was selected to determine permeate fluxes of deionized water at various temperatures under pressures. The temperatures were set as $20^{\circ} \mathrm{C}, 25^{\circ} \mathrm{C}, 30^{\circ} \mathrm{C}$, and $35^{\circ} \mathrm{C}$, respectively. For the given temperature, the pressures were set as $0.15,0.20$, $0.25,0.30$, and $0.35 \mathrm{MPa}$, respectively. According to the collecting data, water permeability $L_{p}$ of the membrane was calculated from (8) by omitting the term of osmotic pressure difference from the permeate flux equation [11] due to the deionized water used. Secondly, three types of flow channel structures including normal flow, spiral flow, and serpentine flow structures were employed in performance experiments. The permeate fluxes and $\mathrm{Mg}^{2+}$ rejections of the membrane were determined for laminar flow, transition flow,

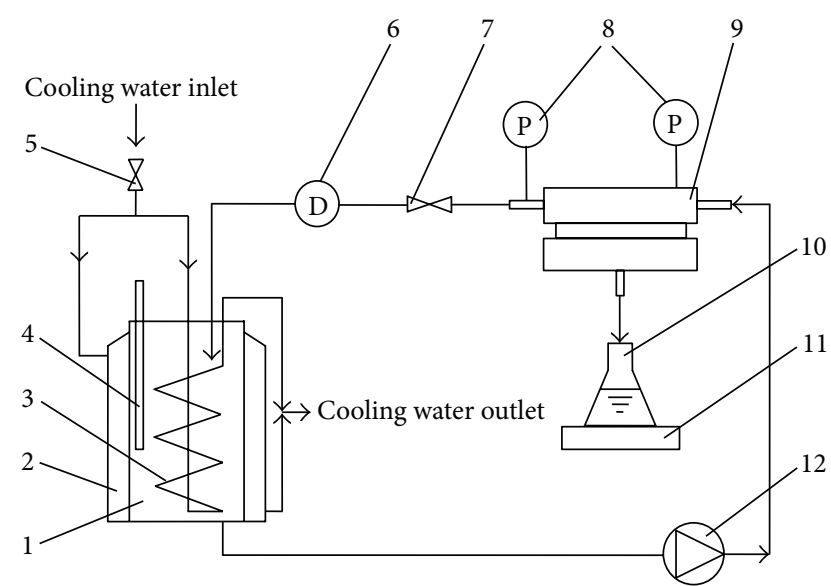

FIGURE 4: The test rig for nanofiltration performance. (1) Feed tank. (2) Coil pipe. (3) Jacket. (4) Thermometer. (5) Cooling water control valve. (6) Glass rotameter. (7) Flow control valve. (8) Pressure gauge. (9) Membrane module. (10) Triangular flask. (11) Electronic balance. (12) Vortex pump.

and turbulent flow under pressure of $0.35 \mathrm{MPa}$ at temperature of $35^{\circ} \mathrm{C}$. Reynolds numbers were of $1200,1400,1600,1800$, and 2000 for laminar flow, of 2500, 2800, 3100, 3400, and 3700 for transition flow, and were of 4000, 4200, 4400, 4600, and 4800 for turbulent flow, respectively:

$$
L_{p}=\frac{\Delta p}{J_{v}},
$$

where $\Delta p$ is the transmembrane pressure drop.

The crossflow rate $q_{V}$ could be calculated from (9) for the spiral or serpentine feed channel module according to Reynolds number:

$$
q_{V}=\frac{\operatorname{Re} \mu A}{\rho d_{h}},
$$

where Re is Reynolds number; $A$ the area of cross section; $d_{h}$ is the hydraulic diameter. In the work, the hydraulic diameter was $11.67 \mathrm{~mm}$ for the spiral feed channel or the serpentine feed channel.

\section{Results and Discussion}

4.1. Water Permeability. Water permeability $L_{p}$ was calculated based on the collecting data in order to investigate the stability of the NF membrane. Based on Hagen-Poiseuille equation, water permeability could also be given by [23]

$$
L_{p}=\frac{r_{p} A_{k}}{8 \mu \Delta x},
$$

where $r_{p}$ is effective membrane pore radius, $A_{k}$ is the membrane porosity, $\Delta x$ is the effective membrane thickness, and $\mu$ is the solvent viscosity.

As shown in Figure $5, L_{p}$ would increase with increasing operating pressure at the given temperature and rise with 


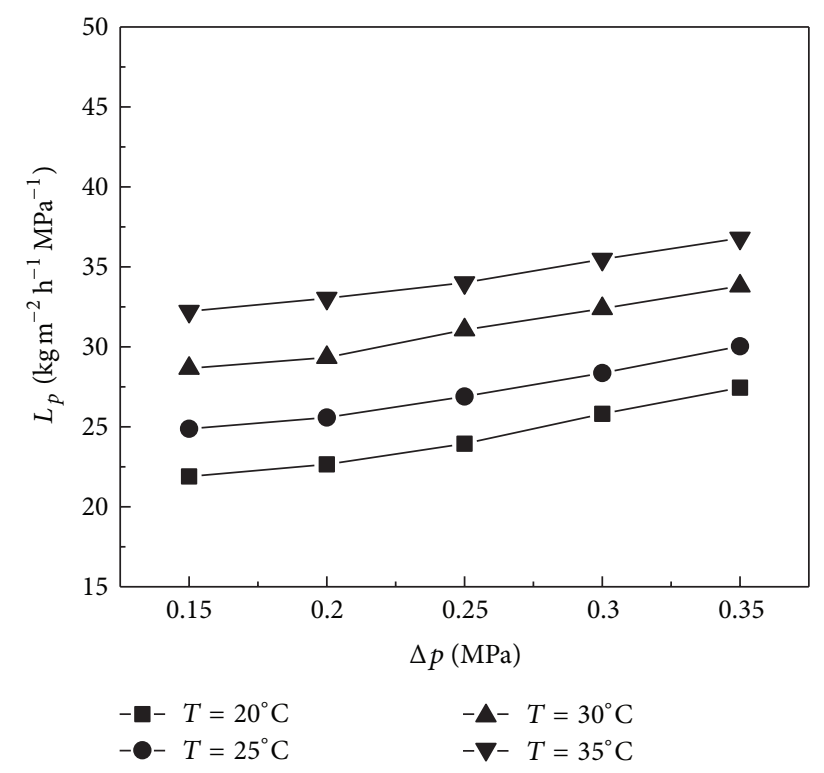

FIGURE 5: Effect of pressure on water permeability at different temperatures.

increasing operating temperature under the given operation pressure. According to Hagen-Poiseuille equation, $L_{p}$ is only correlated to membrane structure parameters $\left(r_{p}, A_{k}\right.$, and $\Delta x$ ) when $\mu$ keeps constant at a given temperature. On the one hand, $\Delta x$ decreased if $r_{p}$ and $A_{k}$ kept constant when pressure increased because membrane was made up of organic polymer materials, which led to increasing $L_{p}$. On the other hand, water viscosity reduced and structure parameters of the membrane did not change when pressure kept constant and temperature rose, which resulted in increasing $L_{p}$.

In a word, water permeability of the polymer NF membrane would vary with the operating pressure and temperature. What is more, the operation temperature and pressure had obvious influences on membrane flux.

4.2. Effect of Reynolds Number. As shown in Figure 6, membrane flux varied slightly with the change of Reynolds number under the given conditions of operating pressure, temperature, and feed concentration, which was in good agreement with the idea that crossflow rate or Re had a little influence on membrane flux presented by Timmer et al. [24]. Meanwhile, Zhu and coworkers also concluded that membrane flux depended hardly on crossflow rate or Re number [25].

The flow pattern of the fluid varied from laminar flow to transition flow or even turbulence flow when Reynolds number increased due to the increase of crossflow rate. On the one hand, the curved flow channel structure hindered the concentration polarization development and the fouling of a membrane, which was helpful to improve membrane flux. On the other hand, the friction loss increased, pressure drop through the module became larger, and filtration driving force decreased with increasing crossflow rate, which went against to the above effect of increasing membrane flux. For

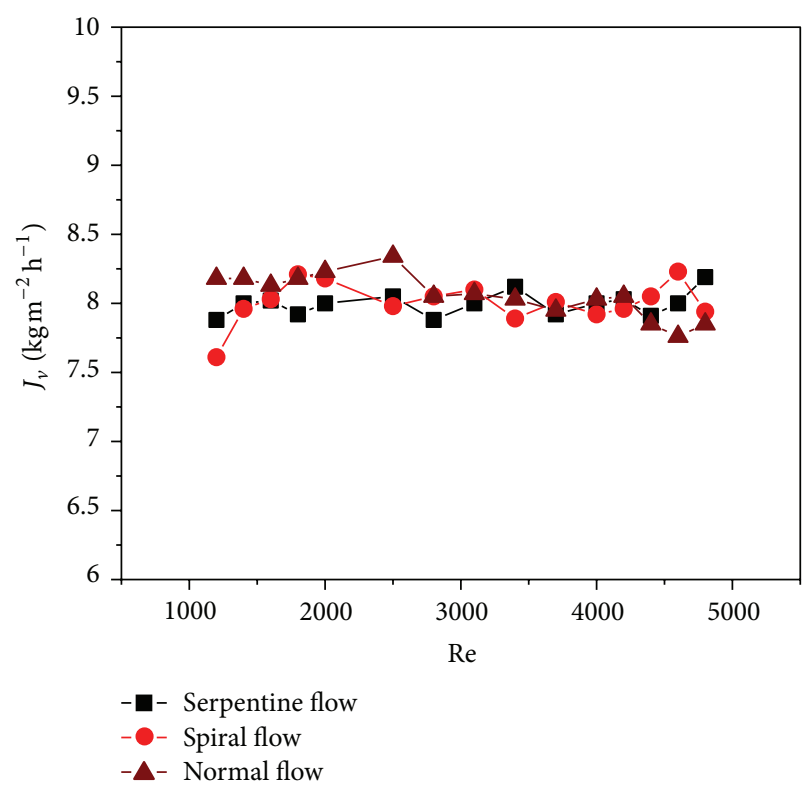

FIgURE 6: Effect of Reynolds number on flux.

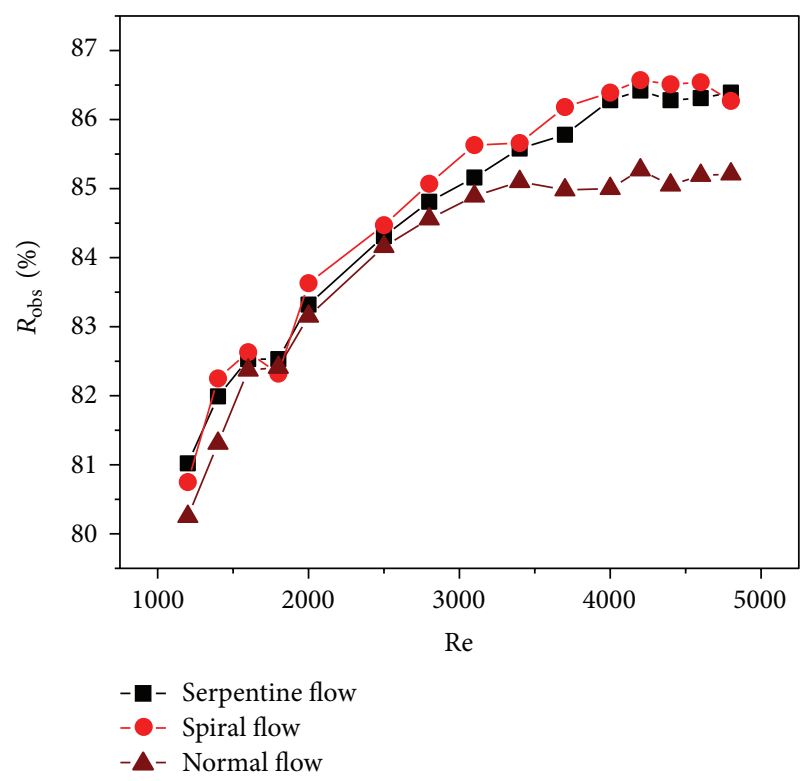

FIGURE 7: Effect of Reynolds number on $\mathrm{Mg}^{2+}$ rejection.

their comprehensive actions, the membrane flux kept nearly constant.

Figure 7 shows the relation of Reynolds number and $\mathrm{Mg}^{2+}$ rejection. As for the three types of flow channel structures, $\mathrm{Mg}^{2+}$ rejection had a significant increasing trend with the rising Reynolds number, which was in a good agreement with the conclusion that the increase of influent flow made ion rejection rise which is summarized by Liu [26]. It was also shown from the results that the rejection kept almost constant for all modules for turbulent flow so that the Reynolds number might hardly influence the rejection when Re was larger than 4000 under the experimental conditions. 
According to the extended Nernst-Planck equation, permeation flux of solute consists of the diffusion flux, the Donnan potential flux, and the convection flux. In the experiments, the flux caused by Donnan potential almost kept constant due to the low feed $\mathrm{Mg}^{2+}$ concentration; so diffusion and convection played important roles on flux of $\mathrm{Mg}^{2+}$. Convective mass transfer refers to that $\mathrm{Mg}^{2+}$ will be transferred to permeate side when feed solution flows through membrane pore. The flux of feed solution is the main influence factor. The larger the flux is, the stronger the convective mass transfer is and the more $\mathrm{Mg}^{2+}$ was brought through the membrane with the feed solution which results in decreasing rejection, and vice versa, $\mathrm{Mg}^{2+}$ rejection will increase. Diffusion mass transfer refers to that $\mathrm{Mg}^{2+}$ diffuses from feed side to permeate side because of the $\mathrm{Mg}^{2+}$ concentration difference across the membrane. $\mathrm{Mg}^{2+}$ concentration on the membrane surface played the key role. The higher the solute concentration on membrane surface is, the larger the diffusion transfer driving force is and the more $\mathrm{Mg}^{2+}$ would diffuse through membrane pores so that the rejection might decrease, and vice versa, $\mathrm{Mg}^{2+}$ rejection would increase. The solution turbulence would disturb the concentration boundary layer, force the solute to move back to the bulk flow, and finally lower the $\mathrm{Mg}^{2+}$ concentration on the membrane surface, which leads to reducing diffusion mass transfer rate and increasing rejection.

4.3. Effect of Flow Channel Structure. Effect of flow channel structure on membrane flux was also shown in Figure 6. It indicated that membrane flux remained almost unchanged for all three flow channel structures at different Re numbers. The flux of the module with normal feed channel was slightly larger for laminar flow. The flux almost kept the same for all the flow channel structures for transition flow. The flux of the module with spiral feed channel became slightly great for turbulent flow. The secondary flows generated at the spiral or serpentine feed channel with the increasing Reynolds number so that the wall shear stress might become higher, the concentration polarization might then be reduced, and the filtration driving force could be increased. Therefore, fluxes for these two feed channels were larger than that for the normal feed channel.

In the experiments, operation pressure was $0.35 \mathrm{MPa}$, and solute concentration was very low, so the osmotic pressure might be relatively low, and effects of flow channel structure on the osmotic pressure could be neglected. Therefore, the varying range of effective filtration driving force was small, and the variation of flux was not obvious. It seems that the flux was independent of the flow channel structure.

It was also shown from Figure 7 that as for laminar flow $\mathrm{Mg}^{2+}$ rejection for the spiral feed channel was almost the same as that for the serpentine feed channel, but both of them were slightly larger than that for the normal feed channel; as for transition flow, $\mathrm{Mg}^{2+}$ rejection for the spiral feed channel was larger than that for the serpentine flow channel, and for the serpentine feed channel it was greater than that for the normal feed channel; as for turbulent flow, $\mathrm{Mg}^{2+}$ rejection for spiral feed channel was also the same as that for serpentine feed channel, but both of them were much larger than that for the normal feed channel. Under the experimental conditions and in varying range of selected Reynolds number, the module with the spiral feed channel had the best $\mathrm{Mg}^{2+}$ rejecting ability, the module with the serpentine feed channel took a second place, and the module with the normal feed channel was the most poor because the Dean vortex on membrane surface made the flow turbulent, hence, reduced $\mathrm{Mg}^{2+}$ concentration on the membrane wall followed by decreasing transmembrane concentration difference, which led to the decreasing driving force of diffusive mass transfer and the rising $\mathrm{Mg}^{2+}$ rejection due to the almost unchanged convective mass transfer rate for all the feed channels.

\subsection{Results of Numerical Simulation}

4.4.1. Velocity Distribution in the Channel. The turbulent flow for the each flow channel was simulated by a CFD method. It is convenient to show the secondary flow by plotting a two-dimensional (2D) velocity field in the given cross section. For spiral feed channel, velocity vector fields in the six given cross sections are shown in Figure 8. Each velocity vector field in the section could be observed along the flow direction. The top, left, or right side of the cross section represents solid wall, and the bottom side stands for membrane wall. It was apparent that secondary flow phenomenon or Dean vortex occurred and shear velocity at membrane wall was considerably large. The results showed that secondary flow generated due to the centrifugal force that worked on fluid, which enhanced the turbulence of fluid and made fluid unstable. Moreover, the large shear velocity nearby membrane wall was able to disturb boundary layer flow and improved shear stress, which hindered the concentration polarization development and the membrane fouling.

Figure 9 showed the velocity vector fields in the given cross sections of the serpentine feed channel. The section velocity vector fields could be observed along the flow direction, and bottom side also represented membrane wall. The secondary flows generated in the 6 cross sections were quite different to a great extent. The shear velocity on the membrane surface in downstream near the U-shaped duct was larger than that in the other zone. The closer to the Ushaped duct the zone was, the stronger the secondary flow was, and vice versa, it became weaker. The shear velocity nearby membrane wall was larger close to the U-shaped duct and became smaller far away from it due to the action of relatively large centrifugal force on the fluid near it. It indicated that fluid layer was strongly disturbed near the membrane wall close to the U-shaped duct, which resulted in strong turbulence.

4.4.2. Shear Stress on Membrane Wall. As the turbulence of fluid became stronger, the shear stress on membrane surface was larger, which was beneficial to destroy the concentration polarization layer and hinder the membrane fouling. Data treatment was carried out for spiral, serpentine, and normal 


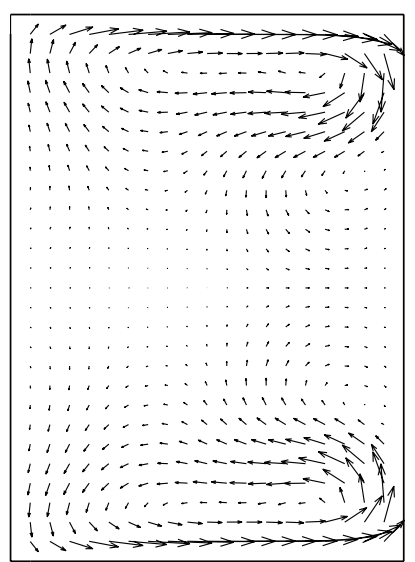

(a) Section 1

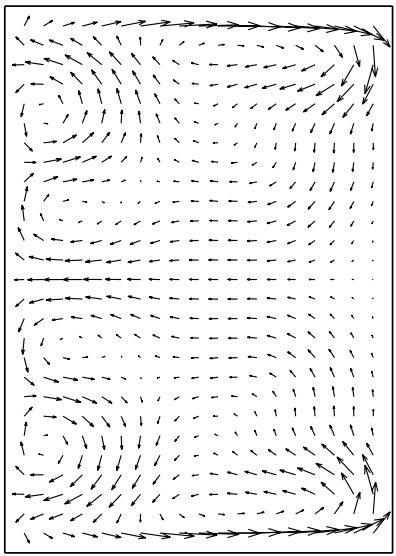

(d) Section 4

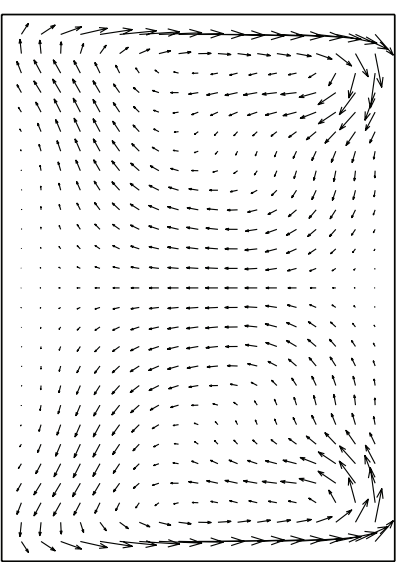

(b) Section 2

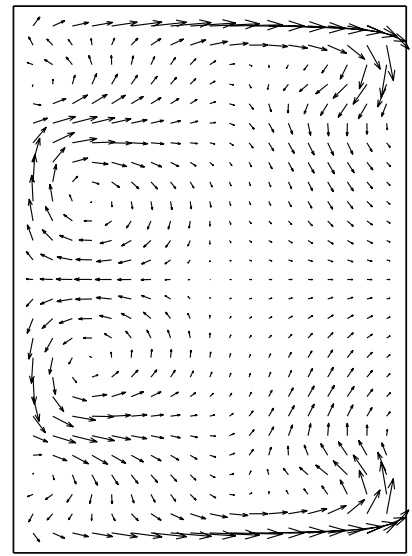

(e) Section 5

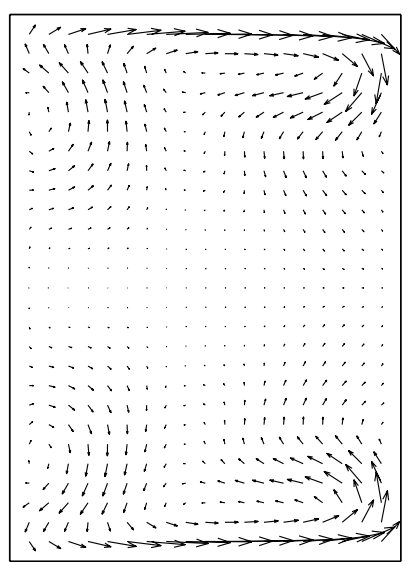

(c) Section 3

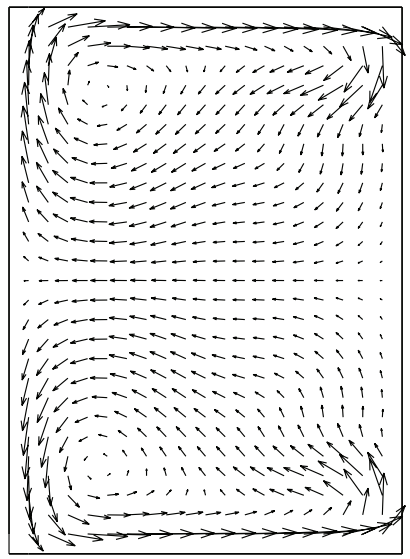

(f) Section 6

FIGURE 8: The velocity vectors in the given cross sections for the spiral feed channel.

feed channels, and the contour map of the shear stress on membrane wall was plotted for each type of flow channel structure. As mentioned in Section 2.4, the inlet velocities for the three types of feed channels were determined differently in order to keep fluid Reynolds number constant so that the effect of inlet velocity on wall shear stress near the inlet might be great. Hence, the wall shear stress field to be observed is far away from the inlet.

The local shear stress on the membrane surface for the spiral feed channel was almost the same, and the shear stress greater than $1.2 \mathrm{~Pa}$ was in relatively small area as shown in Figure 10. Its average wall shear stress was about $0.80 \mathrm{~Pa}$ that is larger than $0.633 \mathrm{~Pa}$ on the wall of inlet pipe due to the effect of the secondary flow. As for the serpentine feed channel, the local shear stress greater than $0.8 \mathrm{~Pa}$ covered a large proportion of the membrane surface, the largest or larger than $1.6 \mathrm{~Pa}$ stress was near the turning point due to the effect of the secondary flow, and the smallest or smaller than $0.4 \mathrm{~Pa}$ stress was in the area at dead corner as shown in Figure 11. The average wall shear stress is about $0.76 \mathrm{~Pa}$, a little bit smaller than that for the spiral feed channel. For the normal feed channel whose inlet velocity was $0.707 \mathrm{~m} / \mathrm{s}$, the local shear stress on membrane wall was relatively large, and flow disturbance was considerably strong in a small area near the inlet, and in a relatively large proportion of the membrane surface, the shear stress was small and even smaller than $0.4 \mathrm{~Pa}$ as shown in Figure 12. Its average wall shear stress was about $0.56 \mathrm{~Pa}$ which was very much smaller than the stress of $2.4 \mathrm{~Pa}$ at the entry of the cell because there was not any effect of the secondary flow in the feed channel. It was also less than the average wall shear stress of the membrane module with any other feed channel.

Generally speaking, shear velocity near the membrane surface increased due to the curved feed channel, which led to increasing wall shear stress, destroyed concentration polarization layer, and low diffusive transport of the solute across the membrane. For the module with spiral or serpentine feed channel, the crossflow rate, necessary to make Re number large, was less than that for the module with normal feed channel so that the latter might consume greater deal of energy than the former in order to get the same average wall shear stress. It indicated that when compared with the other two feed channels, using spiral feed channel would make the shear stress on membrane wall more uniform along the flow channel, destroy the concentration polarization layer, and achieve the high solute rejection. There must have been 


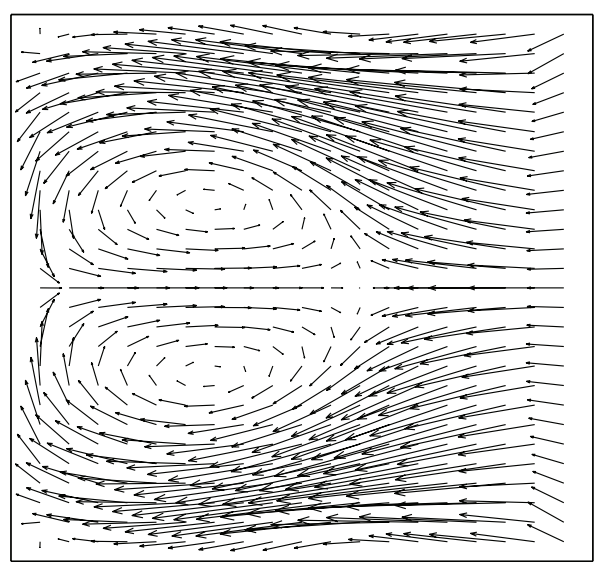

(a) Section 1

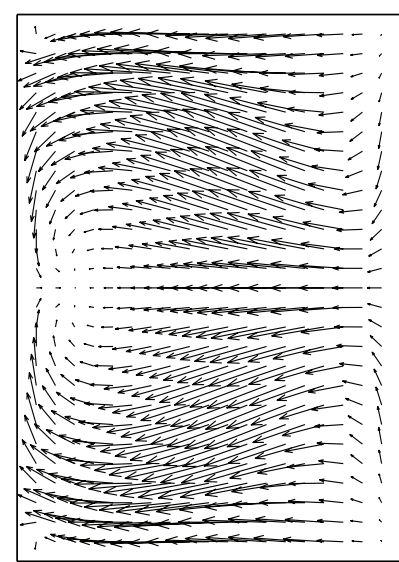

(b) Section 2

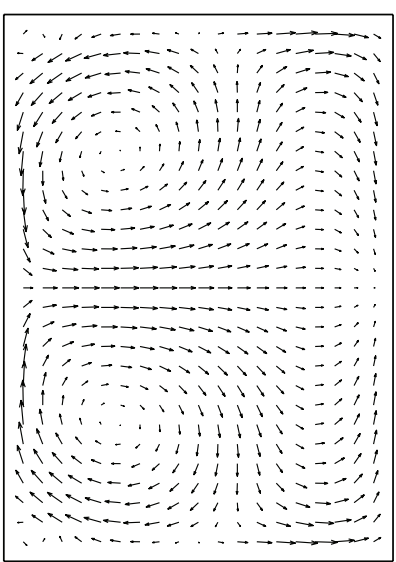

(c) Section 3

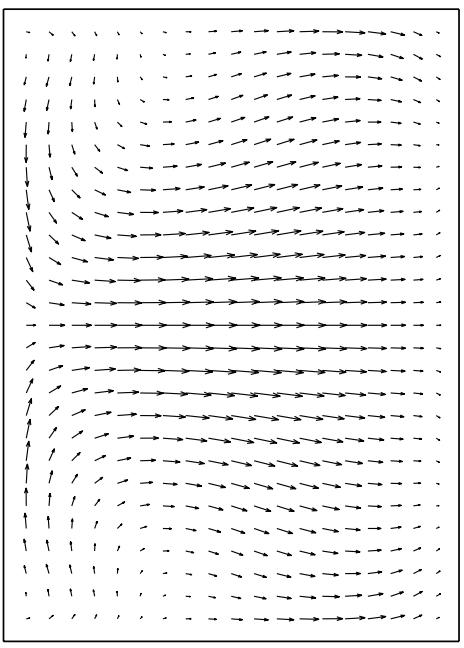

(d) Section 4

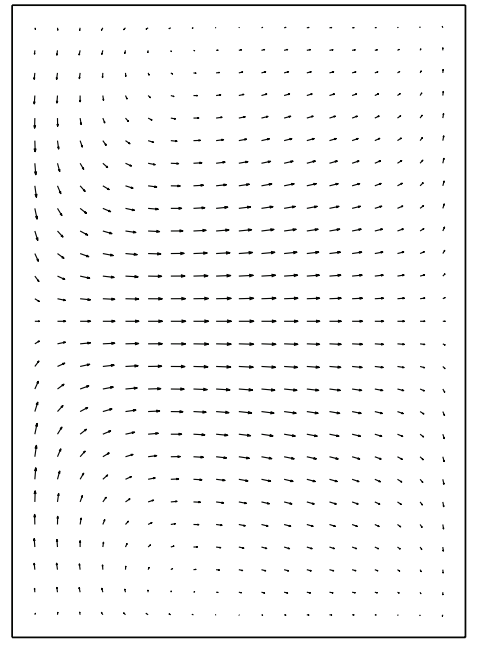

(e) Section 5

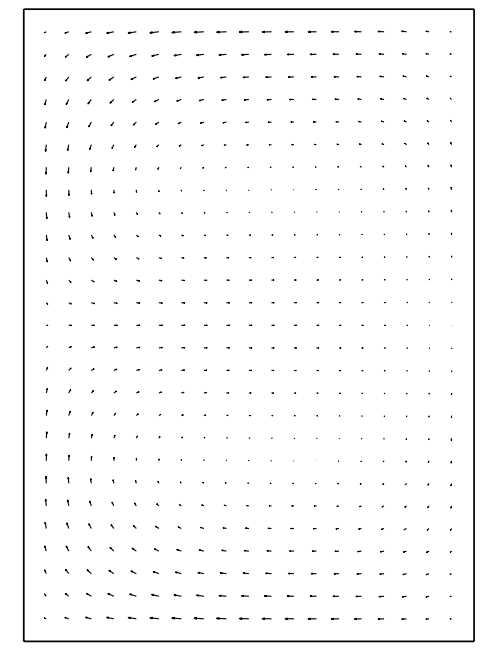

(f) Section 6

FIGURE 9: The velocity vectors in the given cross sections for the serpentine feed channel.

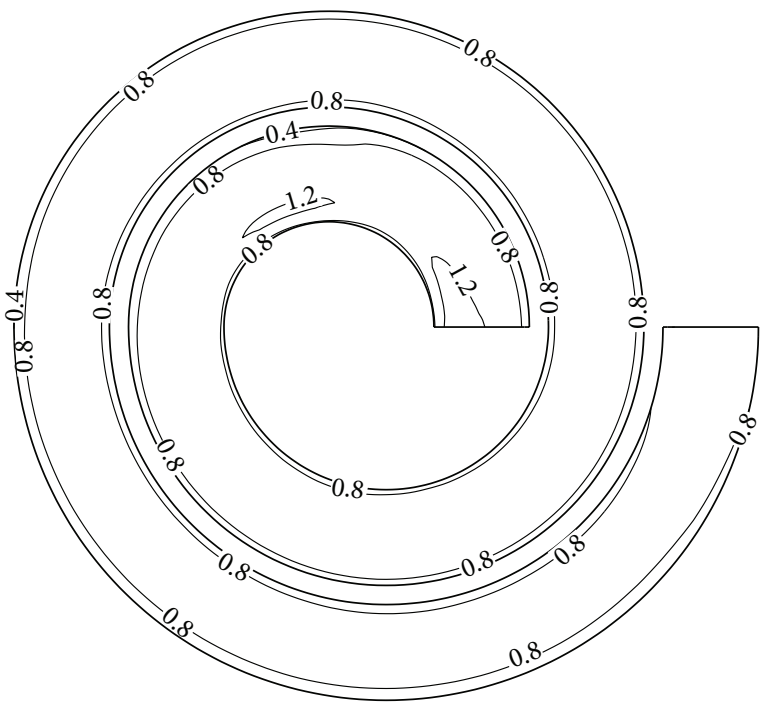

FIGURE 10: The wall shear stress on the membrane surface for the spiral feed channel, Pa.

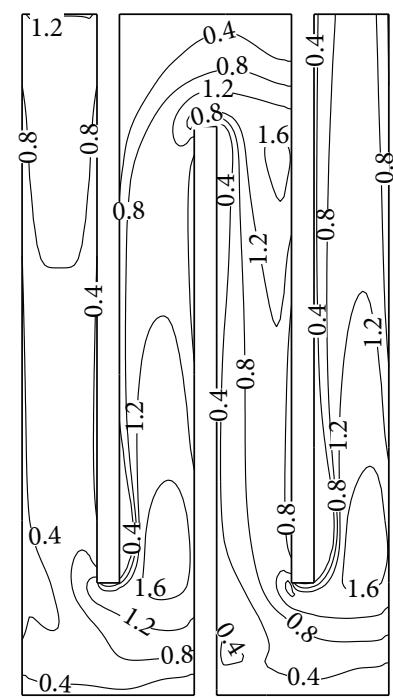

FIGURE 11: The wall shear stress on the membrane surface for the serpentine feed channel, Pa. 


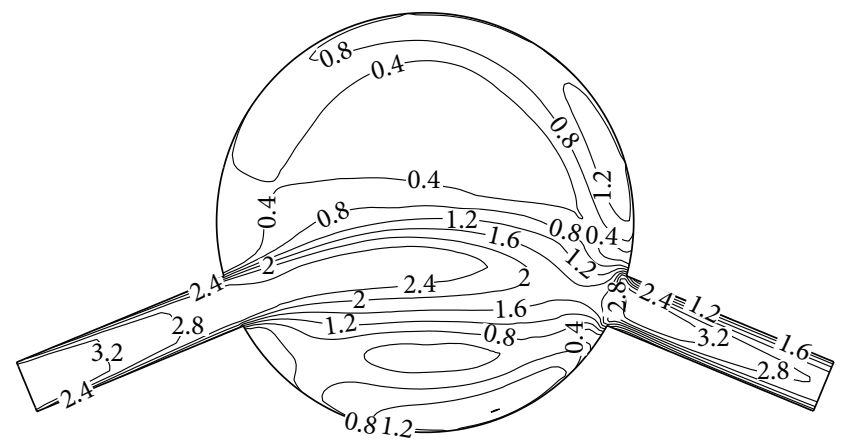

Figure 12: The wall shear stress on the membrane surface for the normal feed channel, $\mathrm{Pa}$.

a critical wall shear stress. The concentration polarization layer would be destroyed and the solute rejection would then keep unchanged when the wall shear stress is greater than it. Thus, using a proper kind of feed channel, for example, the spiral feed channel or serpentine feed channel, could obviously increase the solute retention rate and then improve the separation performance of NF membrane.

\section{Conclusions}

The nanofiltration experiments were carried out using a commercial TFC-SR100 NF membrane and the modules with the normal feed channel and two newly designed feed channels, that is, the spiral and the serpentine feed channels. The experimental results showed that the effect of Reynolds number on flux was not obvious, but the $\mathrm{Mg}^{2+}$ rejection would increase when increasing the Reynolds number. The influence of flow channel structure on flux was not obvious. The effects of the feed channels on the rejection were not significantly at lower Re number. The rejections were almost the same for the spiral feed channel and the serpentine feed channel, but they are larger than that for the normal feed channel. Moreover, the difference between the former and the latter became greater when the Re number was larger than 4000 .

The numerical simulations were also completed at $\mathrm{Re}$ number of 4800 . The results showed that there was secondary flow in the spiral or the serpentine feed channel, which increased the shear stress on the membrane wall and destroyed the concentration polarization layer followed by increasing rejection. The wall shear stress was almost homogeneous along the flow channel for the module with the spiral feed channel. However, the wall stress was relatively low for the module with normal feed channel since there was not any secondary flow in the cell. It suggested that the spiral flow channel structure was a proper one to be used to improve the filtration performance of the NF membrane.

\section{References}

[1] P. Eriksson, "Nanofiltration extends the range of membrane filtration," Environmental Progress, vol. 7, no. 1, pp. 58-62, 1988.
[2] X. L. Wang, W. J. Shang, D. X. Wang, L. Wu, and C. H. Tu, "Characterization and applications of nanofiltration membranes: state of the art," Desalination, vol. 236, no. 1-3, pp. 316-326, 2009.

[3] A. I. Schafer, A. G. Fane, and T. D. Waite, Nanofiltration: Principles and Applications, Elsevier Science, 2004.

[4] C. Ba, J. Langer, and J. Economy, "Chemical modification of P84 copolyimide membranes by polyethylenimine for nanofiltration," Journal of Membrane Science, vol. 327, no. 1-2, pp. 49$58,2009$.

[5] M. Jahanshahi, A. Rahimpour, and M. Peyravi, "Developing thin film composite poly(piperazine-amide) and poly(vinylalcohol) nanofiltration membranes," Desalination, vol. 257, no. 1-3, pp. 129-136, 2010.

[6] M. N. Abu Seman, M. Khayet, Z. I. Bin Ali, and N. Hilal, "Reduction of nanofiltration membrane fouling by UV-initiated graft polymerization technique," Journal of Membrane Science, vol. 355, no. 1-2, pp. 133-141, 2010.

[7] G. T. Ballet, A. Hafiane, and M. Dhahbi, "Influence of operating conditions on the retention of phosphate in water by nanofiltration," Journal of Membrane Science, vol. 290, no. 1-2, pp. 164-172, 2007.

[8] X. M. Li, D. Wang, T. Chai, B. W. Su, and C. J. Gao, "Study on seawater softening by nanofiltration membrane," Journal of Chemical Engineering of Chinese Universities, vol. 23, no. 4, pp. 582-586, 2009.

[9] J. Z. Pang, J. Y. Li, Q. Wang, and Z. Z. Yang, "Study on treatment of salt chemical industry wastewater with nanofiltration membrane," Journal of Tianjin Polytechnic University, vol. 29, no. 5, pp. 6-9, 2010.

[10] S. Darvishmanesh, A. Buekenhoudt, J. Degrève, and B. van der Bruggen, "General model for prediction of solvent permeation through organic and inorganic solvent resistant nanofiltration membranes," Journal of Membrane Science, vol. 334, no. 1-2, pp. 43-49, 2009.

[11] F. Fadaei, S. Shirazian, and S. N. Ashrafizadeh, "Mass transfer modeling of ion transport through nanoporous media," Desalination, vol. 281, pp. 325-333, 2011.

[12] X. L. Wang, C. H. Tu, Y. Y. Fang, Y. Xiao, and B. Zhou, “The researches on the pore structure, charge property, separation mechanism and electrokinetic phenomena of nanofiltration membranes," Membrane Science and Technology, vol. 31, no. 3, pp. 127-134, 2011 (Chinese).

[13] J. L. C. Santos, V. Geraldes, S. Velizarov, and J. G. Crespo, "Investigation of flow patterns and mass transfer in membrane module channels filled with flow-aligned spacers using computational fluid dynamics (CFD)," Journal of Membrane Science, vol. 305, no. 1-2, pp. 103-117, 2007.

[14] M. Frappart, M. Y. Jaffrin, L. H. Ding, and V. Espina, "Effect of vibration frequency and membrane shear rate on nanofiltration of diluted milk, using a vibratory dynamic filtration system," Separation and Purification Technology, vol. 62, no. 1, pp. 212221, 2008.

[15] J. S. Vrouwenvelder, C. Picioreanu, J. C. Kruithof, and M. C. M. van Loosdrecht, "Biofouling in spiral wound membrane systems: three-dimensional CFD model based evaluation of experimental data," Journal of Membrane Science, vol. 346, no. 1, pp. 71-85, 2010.

[16] G. A. Fimbres-Weihs and D. E. Wiley, "Review of 3D CFD modeling of flow and mass transfer in narrow spacer-filled channels in membrane modules," Chemical Engineering and Processing, vol. 49, no. 7, pp. 759-781, 2010. 
[17] F. Cortés-Juan, B. Balannec, and T. Renouard, "CFD-assisted design improvement of a bench-scale nanofiltration cell," Separation and Purification Technology, vol. 82, pp. 177-184, 2011.

[18] H. H. Zhan, H. Zhu, J. D. Chen, and G. Wang, "Numerical simulation of secondary Flow (Dean vortices) in $90^{\circ}$ curved tube," Boiler Technology, vol. 41, no. 4, pp. 1-5, 2010.

[19] W. R. Dean, "Fluid motion in a curved channel," pp. 403-419, 1928, http://rspa.royalsocietypublishing.org/.

[20] T. H. Shih, W. W. Liou, A. Shabbir, Z. Yang, and J. Zhu, "A new $k-\varepsilon$ eddy viscosity model for high reynolds number turbulent flows," Computers and Fluids, vol. 24, no. 3, pp. 227-238, 1995.

[21] M. Lateb, C. Masson, T. Stathopoulos, and C. Bédard, "Comparison of various types of $k-\varepsilon$ models for pollutant emissions around a two-building configuration," Journal of Wind Engineering and Industrial Aerodynamics, vol. 115, pp. 9-12, 2013.

[22] D. M. Wang, Fenxihuaxueshiyan, Huazhong University of Science and Technology Press, Wuhan, China, 2007.

[23] J. H. Ma, B. H. Sun, J. Zhou, and Q. Li, "Study of separation technology by using nanofiltration membrane in seawater," Journal of Salt and Chemical Industry, vol. 36, no. 5, pp. 1-5, 2007 (Chinese).

[24] J. M. K. Timmer, H. C. van der Horst, and T. Robbertsen, "Transport of lactic acid through reverse osmosis and nanofiltration membranes," Journal of Membrane Science, vol. 85, no. 2 , pp. 205-216, 1993.

[25] A. N. Zhu, W. P. Zhu, and Y. C. Zhang, "Fouling problems of nanofiltration and influence factors of nanofiltration membrane performance," Membrane Science and Technology, vol. 23, no. 1, pp. 43-49, 2003.

[26] D. J. Liu, Study on seawater nanofiltration [M.S. thesis], Tianjin University, 2008. 

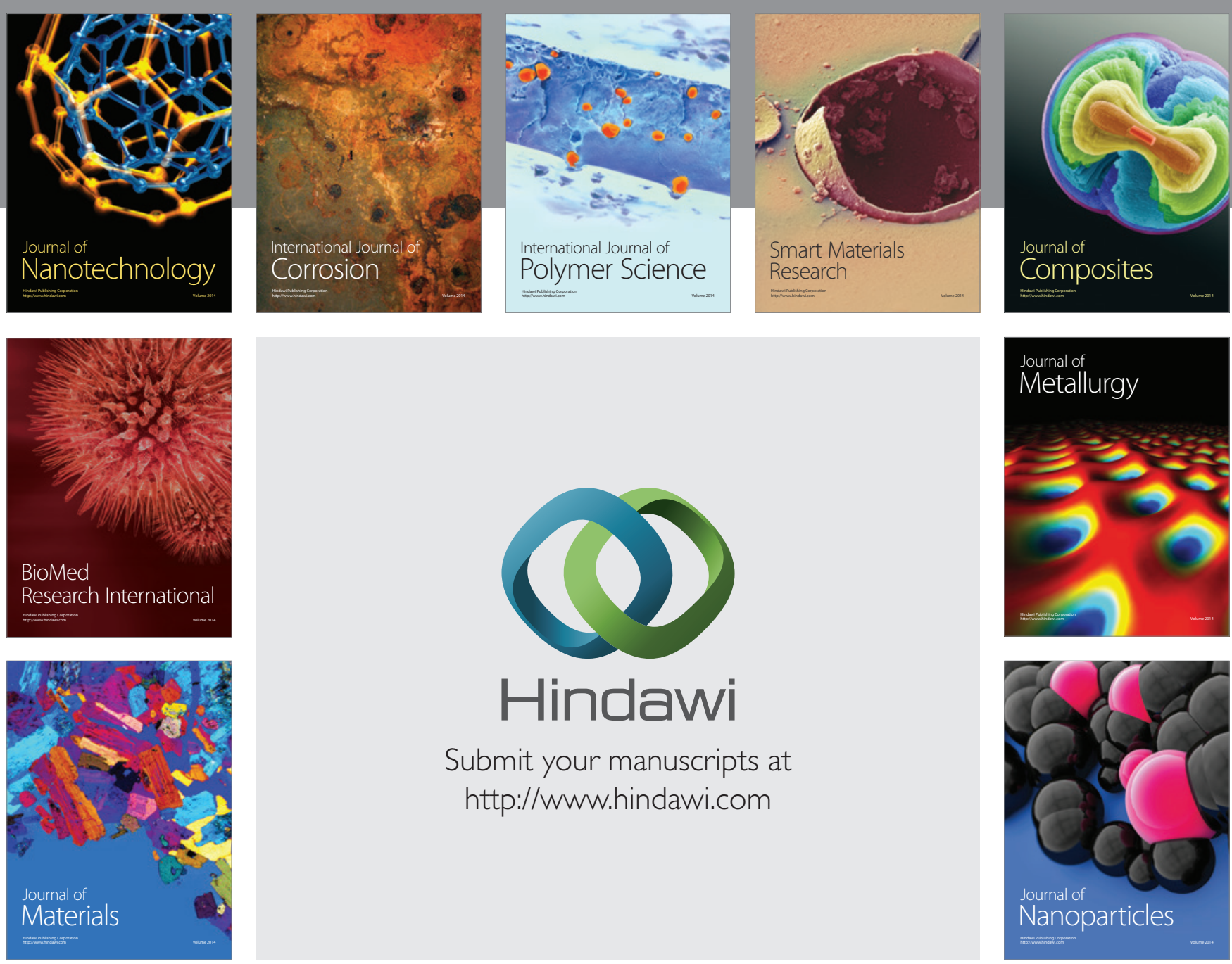

Submit your manuscripts at http://www.hindawi.com
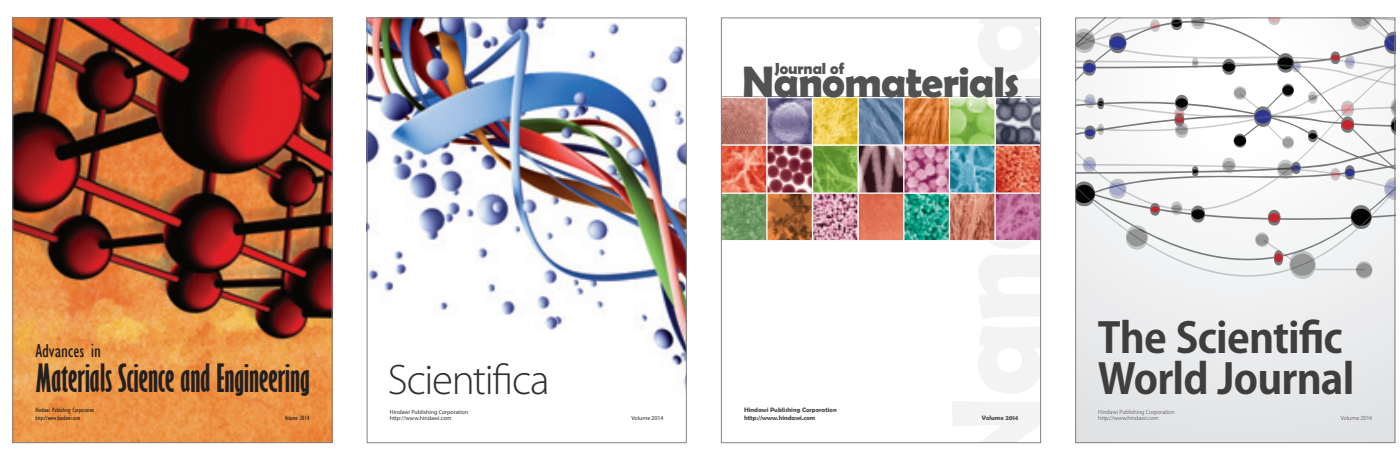

\section{The Scientific World Journal}
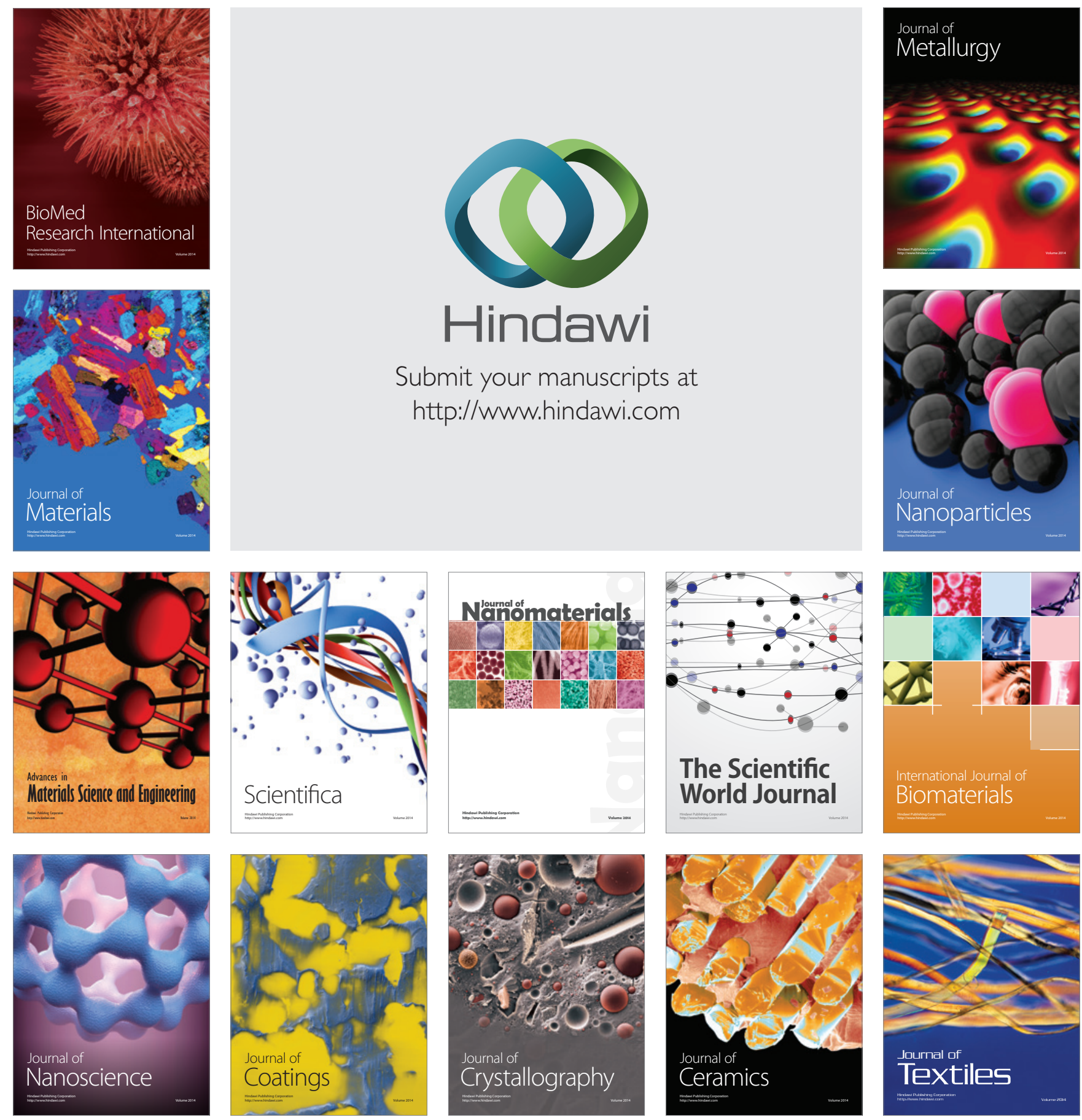